\title{
Ensuring compliance in hemodynamic outcomes
}

\author{
S. Ram Kumar, MD, PhD, FACS
}

\footnotetext{
From the Division of Cardiac Surgery, Department of Surgery, University of Southern California, and Heart Institute, Children's Hospital of Los Angeles, Los Angeles, Calif.

Disclosures: Author has nothing to disclose with regard to commercial support.

Received for publication April 14, 2016; accepted for publication April 15, 2016; available ahead of print May 24, 2016.

Address for reprints: S. Ram Kumar, MD, PhD, FACS, Children's Hospital of Los Angeles, 4650 Sunset Blvd, Mailstop \#66, Los Angeles, CA 90033 (E-mail: rsubramanyan@ chla.usc.edu).

J Thorac Cardiovasc Surg 2016;152:514-5

$0022-5223 / \$ 36.00$

Copyright $(\subset 2016$ by The American Association for Thoracic Surgery

http://dx.doi.org/10.1016/j.jtcvs.2016.04.065
}

Ventricular septal defect (VSD) (Figure 1) continues to be among the most commonly repaired congenital heart defects. Its pathophysiology and the influence of repair are generally well understood based on clinical observations, noninvasive monitoring and intuitive extrapolation of predicted physiology. Muneuchi and colleagues ${ }^{1}$ have provided definitive hemodynamic data obtained from pre- and postoperative catheterization in 100 patients with isolated VSD who underwent successful closure over a 15-year period. For the most part, their data reaffirm the anticipated clinical outcome: following elimination of a large left-toright shunt, there is an immediate and significant reduction in pulmonary pressures.

The authors ${ }^{1}$ leveraged their unique institutional practice pattern, which includes routine catheterization before and immediately following VSD repair, for the analyses

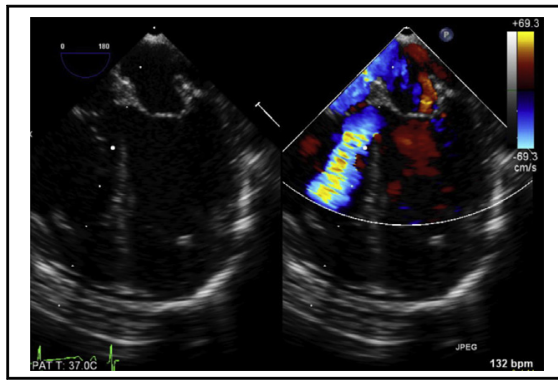

Transesophageal echocardiogram showing a significant ventricular septal defect.

\section{Central Message}

Extensive catheter-based physiologic data reaffirm anticipated clinical outcomes in children undergoing ventricular septal defect repair.

See Article page 507.

reported in this article. There are clinical features that also appear to be unique to their patient cohort. Repair was undertaken at a median age of 3 months (including $20 \%$ with outlet VSDs), and there were rather unusually large left-to-right shunts (some with Qp:Qs > 5). The authors chose a very conservative definition of pulmonary

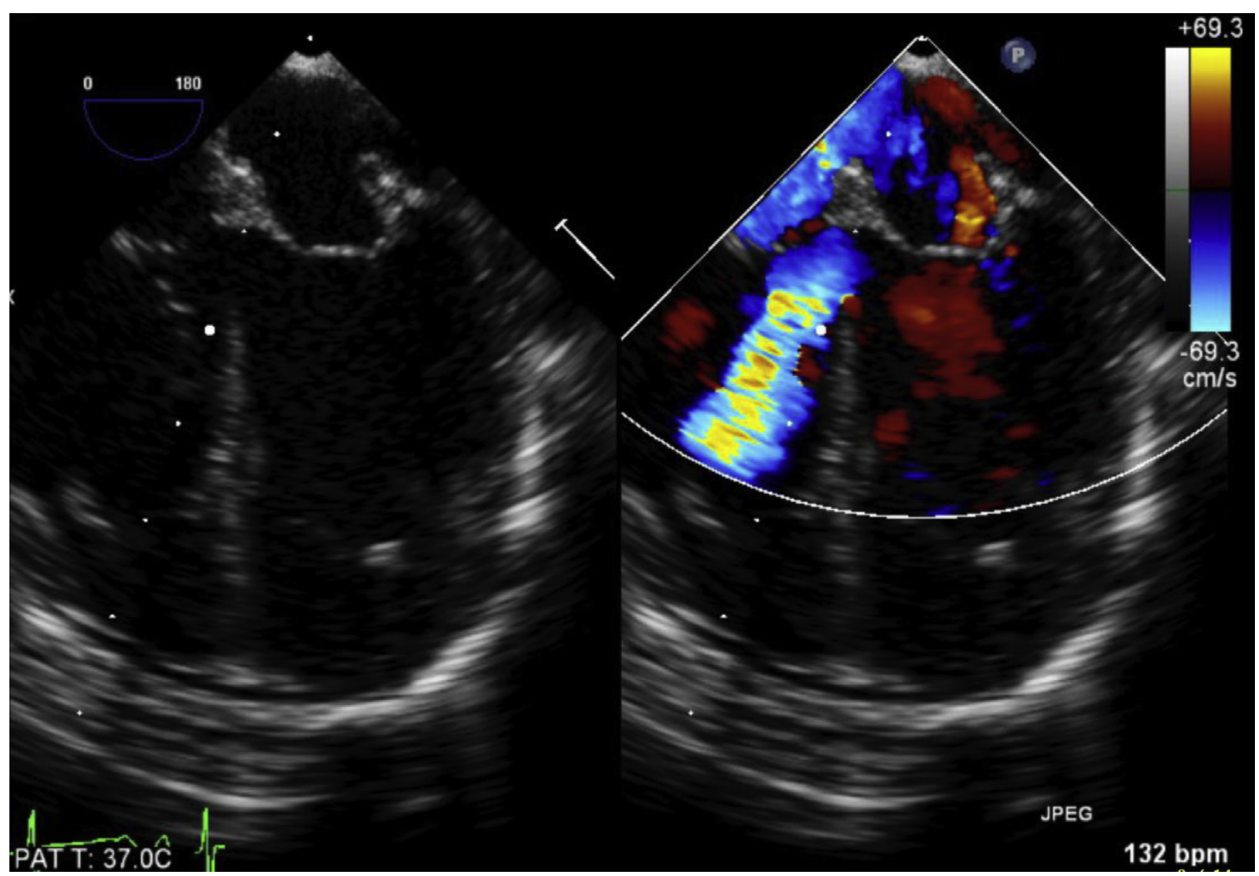

FIGURE 1. Transesophageal echocardiogram showing membranous ventricular septal defect with significant left-to-right shunting. 
hypertension: a mean pressure $>25$ Torr or pulmonary to systemic pressure ratio $>0.3$. Interestingly, pulmonary vasoreactivity was not tested and no vasodilator therapy was used for a prolonged period of time. These factors may limit the wider application of their results. Regardless, the authors need to be commended for validating a clinical hunch with real data and for providing such extensive catheter-based physiologic data that are both unique and valuable.

We have all learned to accept calculated hemodynamic variables in small children undergoing catheterization with a grain of salt. Do we really need another calculated variable, pulmonary compliance $(\mathrm{Cp})$, to make the right clinical decision in children with VSD? Statistically, lower preoperative $\mathrm{Cp}$ was independently associated in this study with higher postoperative pulmonary pressures. However, postoperative hemodynamic parameters were measured 13 days after surgical repair. It is difficult to imagine why pulmonary vasculature would become significantly less compliant immediately after a VSD is closed. Is the more germane explanation that the mathematical equation to derive $\mathrm{Cp}$ was more heavily influenced by the major drop in pulmonary blood flow resulting from elimination of the shunt? Is it the case that what really influenced the postoperative pulmonary pressures was in fact the severity of the preoperative left-to-right shunt? The logistics of how the regression model was set up by the authors (such as using interdependent parameters simultaneously as independent variables) may have resulted in 1 parameter being more significant than another. Also, as acknowledged by the authors, it is plausible that remodeling of the pulmonary vascular bed continues well beyond this early postoperative evaluation point, ${ }^{1}$ and their study does not address the true natural history of the disease process.

The real clinical dilemma relates to patients with high pulmonary pressure and resistance (and low compliance) in the setting of a left-to-right shunt. Unfortunately, Muneuchi and colleagues ${ }^{1}$ do not offer us any insight into this problem. Whereas the compliance and resistance curves do shift with shunt elimination, the patient-specific changes in resistance or compliance plots are more horizontal to the right of the graph in the authors' Figure $2, B,{ }^{1}$ and the $\log$ arithmic transformed correlation lines in their Figure 2, $C$, approach one another with increasing resistance. This implies that the purported postoperative hemodynamic changes in the pulmonary vasculature are less pronounced in children with high preoperative pulmonary resistance and low compliance. It is this subset of patients who require more stringent evaluation in future studies.

\section{Reference}

1. Muneuchi J, Nagatomo Y, Watanabe M, Joo K, Onzuka T, Ochiai Y, et al. Relationship between pulmonary arterial resistance and compliance among patients with pulmonary arterial hypertension and congenital heart disease. J Thorac Cardiovasc Surg. 2016;152:507-13. 